\title{
¿Qué informa la prensa escrita argentina acerca del fenómeno de cyberbullying?
}

\author{
What does the argentine written press report about \\ the phenomenon of cyberbullying?
}

Alejandra Inés Lanzillotti ${ }^{1}$ y Guido Pablo Korman ${ }^{2}$

1Licenciada en Psicología. Becaria Doctoral del Consejo Nacional de Investigaciones Científicas y Técnicas (CONICET) en el Centro Argentino de Etnología Americana (CAEA). Jefa de Trabajos Prácticos en la segunda Cátedra de Estadística de la carrera de Licenciatura en Psicología de la Facultad de Psicología de la Universidad de Buenos Aires (UBA), Argentina.

E-mail: alejandralanzillotti@psi.uba.ar

2Doctor en Psicología. Investigador Adjunto del Consejo Nacional de Investigaciones Científicas y Técnicas (CONICET) en el Centro Argentino de Etnología Americana (CAEA). Profesor Adjunto en la segunda cátedra de Clínica Psicológica y Psicoterapias: Psicoterapias, Emergencia e Interconsultas de la carrera de Licenciatura en Psicología de la Facultad de Psicología de la Universidad de Buenos Aires (UBA), Argentina. Docente de posgrado en la Facultad de Psicología de la UBA. E-mail: gkorman@psi.uba.ar

Centro Argentino de Etnología Americana (CAEA-CONICET). Consejo Nacional de Investigaciones Científicas y Técnicas (CONICET).

Ciudad Autónoma de Buenos Aires, Argentina.

\section{Resumen}

En los últimos años, los términos bullying y cyberbullying se han ido instalando en los medios de comunicación argentinos, entre ellos en la prensa escrita. Se trata de términos técnicos, ya que hacen referencia a problemáticas específicas, que han sido adoptados por la prensa local a fin de dar cuenta, principalmente, de las características, consecuencias y posibles abordajes de estos fenómenos. Teniendo en cuenta que la lectura y análisis de aquello que publica la prensa respecto de un fenómeno permitiría observar aspectos interesantes del proceso de difusión de la información, del discurso empleado y del sentido colectivo que le otorga, el objetivo general de este trabajo se orientó a indagar el tipo de información y su contenido con respecto al fenómeno de cyberbullying en la prensa escrita argentina. Se discuten los hallazgos obtenidos mediante el empleo de un análisis temático y de contenido a fin de ampliar el conocimiento sobre este fenómeno en nuestro medio local.

Palabras clave: maltrato escolar; cyberbullying; ciberbullying; medios de comunicación social; prensa escrita; enfoque cualitativo.

\section{Abstract}

In recent years, the terms "bullying" and "cyberbullying" have been adopted by the Argentinian media, including the written press. These are technical terms that refer to specific problems. The local press has adopted them in order to report about the characteristics, consequences, and possible approaches 
to these situations. The analysis of the press' publications on a certain phenomenon enables us to see interesting aspects of the process of dissemination of information, the discourse used and the collective consciousness it creates. Therefore, the general objective of this work is focused on investigating the type of information and contents regarding cyberbullying in the Argentinian press. A qualitative methodological approach was used, which included the use of a thematic and content analysis. It is noted that only a low proportion of journalism articles was found in which the three basic characteristics of the cyberbullying were pointed out (the intentionality, the repetition in time and the existence of an imbalance of power). In addition, it is observed that the written press provides more information related to the characteristics and possible approaches to cyberbullying, and, to a lesser extent, to its detection. The negative consequences of this problem regarding the psychological health of those involved are mentioned, highlighting indicators and individual consequences, but without referring to indicators or to collective, institutional or social consequences. The development of a hostile and unsupportive school environment, the normalization of aggressive behaviors, the positive assessment of violent behavior in order to obtain social success, and the possible consequences for teachers and families are not mentioned by the press. Also, it is observed that the press gives little room to the students' point of view, who, from their experiences, attitudes and perspectives on this phenomenon, could make important contributions for the development of both prevention strategies and effective boarding tools. The findings obtained are discussed through thematic and content analysis in order to broaden the knowledge about this phenomenon in our local environment. Within the limitations of this study, it is worth mentioning that the different publications began the digitization of their copies in different years, which could affect the total number of articles surveyed and the amount registered for each of the digital portals. Likewise, for this study, only two descriptors were used to carry out the search, so future studies could incorporate other terms in order to broaden and deepen the findings. This could also contribute to generate other analysis categories, in order to record other aspects related to the type and content of information not addressed in this study.

Keywords: school abuse; cyberbullying; bullying; social media; written press; qualitative approach.

\section{Introducción}

El maltrato entre estudiantes dentro de la escuela constituye una problemática compleja que viene siendo objeto de interés académico y social en los últimos años debido a las consecuencias nocivas que ocasiona tanto a nivel individual como grupal, institucional y comunitario (Mason, 2008; Ortega-Ruiz, 1994). La escuela constituye uno de los primeros espacios de socialización en donde el grupo de pares adquiere una gran relevancia y significación en la vida de niños, niñas y adolescentes (Ayestarán-Etxeberria, 1987). En ocasiones, dentro de la dinámica grupal pueden surgir formas de maltrato y hostigamiento que repercuten negativamente tanto en los estudiantes involucrados de manera directa como también en todo el grupo de iguales, afectando el clima escolar y el mantenimiento de una convivencia respetuosa (Ortega-Ruiz, 1994, 2010). Cuando un/a estudiante o grupo de ellos/as, de forma hostil y abusando de un poder real o ficticio, lleva a cabo una serie de acciones negativas contra un/a compañero/a con la intención de causarle un daño, y estas acciones se producen de manera repetida y de forma prolongada en el tiempo, tiene lugar lo que se conoce como maltrato escolar o bullying (Olweus, 1998). El maltrato escolar constituye un grave problema dentro de la dinámica de pares, ya que implica una disfuncionalidad a nivel del grupo de coetáneos (Resett, Costa, Murata y Falcone, 2015). Es decir, se trata de una forma especí- 
fica de violencia entre los/las alumnos/as que involucra una dinámica compleja en el marco de sus relaciones interpersonales, en la que la instalación de una relación asimétrica de poder produce la ruptura de la esperable paridad entre los iguales (Monelos, Mendiri y GarcíaFuentes, 2015; Olweus, 1998; Ortega-Ruiz, 1994; Ortega-Ruiz y Mora-Merchan, 2008).

En los últimos años, el creciente avance tecnológico, las amplias posibilidades de accesibilidad y portabilidad y el uso cada vez más temprano de tecnologías de comunicación electrónica entre la población infanto-juvenil han posibilitado el surgimiento de una nueva modalidad de maltrato escolar denominada cyberbullying (Aboujaoude, Savage, Starcevic y Salame, 2015; Li, 2006; Tokunaga, 2010). El cyberbullying (en lo que sigue CB) puede definirse como un tipo de conducta intencional y agresiva, que se repite de forma frecuente en el tiempo, a través del uso de dispositivos electrónicos por parte de un individuo o grupo sobre una persona que no puede defenderse fácilmente por sí misma (Smith, Mahdavi, Carvalho, Fisher, Russell y Tippett, 2008). El envío reiterado de mensajes desagradables, ofensivos y/o insultantes (hostigamiento) o de amenazas de daño (acecho); la difusión de contenidos difamatorios, rumores o mentiras para dañar la reputación (denigración), de información privada de una persona sin su consentimiento (violación de la intimidad) o de videos en los que se registra una agresión física a fin de humillar a la víctima (happy slapping) son algunas de las formas en que el CB puede ser ejercido. Otras modalidades incluyen el engaño para sonsacar información privada a una persona para luego enviarla y/o difundirla de manera malintencionada (sonsacamiento y revelación de información), la exclusión deliberada de sitios o grupos en línea (exclusión) y el robo de contraseñas y la creación de perfiles falsos para enviar mensajes o hacer comentarios maliciosos en nombre de otra persona (suplantación) (Bauman, 2015; Kowalski, Limber y Agatston, 2012; Shaw, 2005; Willard, 2005, 2007).
En cuanto a sus características, se observa cierto consenso en señalar que el CB comparte los componentes básicos del bullying tradicional $-\mathrm{o}$ cara a cara-: la intencionalidad, la repetición en el tiempo y la existencia de un desequilibrio de poder o relación asimétrica (Olweus y Limber, 2017; Smith et al., 2008). Sin embargo, teniendo en cuenta las posibilidades que brinda el ciberespacio, se deben considerar ciertas características particulares del CB como la continuidad en el tiempo - ya que la agresión puede ser ejecutada y recibida en todo momento, cualquier día y en cualquier horario- y la continuidad en el ciberespacio teniendo en cuenta que los contenidos compartidos en la web son imperecederos- (Slonje, Smith y Frisén, 2013). A su vez, la característica relacionada con la repetición en el tiempo puede ser reconsiderada ya que la presencia de un solo episodio de agresión puede entenderse como susceptible de ser repetido si se tiene en cuenta el hecho de que puede difundirse y replicarse ampliamente en el ciberespacio (Dooley, Pyzalski y Cross, 2009; Slonje et al., 2013). Asimismo, el desequilibrio de poder puede vincularse con la posibilidad de anonimato digital (Dehue, Bolman y Vollink, 2008; Patchin e Hinduja, 2006), la mayor habilidad tecnológica de quien realiza la agresión (Kowalski et al., 2012; Slonje et al., 2013) y/o cierta popularidad del agresor en el espacio virtual (Grigg, 2010). Otras particularidades se relacionan con la amplitud de la audiencia, el alcance masivo de la agresión y la posibilidad de que otros puedan participar en ella (Wong-Lo y Bullock, 2014).

En cuanto a las consecuencias del CB, a nivel general, entre los principales efectos que se han observado se destacan la presencia de sintomatología depresiva, sentimientos de soledad, tristeza, miedo, preocupación, frustración e ira, irritabilidad, somatizaciones, trastornos del sueño, problemas de concentración y de rendimiento escolar, síntomas de ansiedad, comportamientos autolíticos e ideación e intentos suicidas (Dooley, Gradinger, Strohmeier, Cross y Spiel, 2010; Erdur-Backer 
y Tanrikulu, 2010; Gámez-Guadix, Orue, Smith y Calvete, 2013; Hinduja y Patchin, 2010; Juvoven y Gross, 2008; Kowalski y Limber, 2013; Ortega-Barón, Torralba y Buelga, 2017; Patchin y Hinduja, 2010; Roberts, Axas, Nesdole y Repetti, 2016; Van Geel, Vedder y Tanilon, 2014; Ybarra, 2004). A su vez, el CB tiene consecuencias perniciosas para todos los implicados ya que los observadores también serían receptores de aprendizajes y hábitos negativos que podrían influir en su comportamiento actual y futuro, vinculados con la normalización de las conductas violentas, la falta de solidaridad, la reducción de la empatía y el aprendizaje de habilidades sociales inadecuadas (Garaigordobil, 2014; Ortega-Ruiz, 2010). Al respecto, se ha hallado que tanto la empatía como las emociones positivas son importantes precursores de las habilidades sociales adecuadas en la niñez y en la adolescencia (Oros y Fontana Nalesso, 2015), por lo que las consecuencias negativas que generan las situaciones de CB podrían aumentar el riesgo de que los implicados sufran desajustes psicosociales y trastornos psicopatológicos en un período del ciclo vital que resulta clave para el desarrollo de distintas habilidades sociales y rasgos de personalidad (Garaigordobil, 2014). Asimismo, el clima escolar e institucional podrían verse afectados y repercutir negativamente no solo en los estudiantes sino también en todos los actores del ámbito educativo, incluyendo a docentes, directivos y padres (Carozzo, Benites, Zapata y Horna, 2012; Mason, 2008).

En lo que refiere a Argentina, si bien aún son muy escasas las investigaciones orientadas a evaluar la prevalencia y las características que el CB adquiere en nuestro contexto, pueden mencionarse algunos datos generales extraídos del estudio realizado por el Fondo de las Naciones Unidas para la Infancia (UNICEF) en el año 2016. De acuerdo con lo relevado, aproximadamente el $43 \%$ de los adolescentes encuestados informó haber recibido mensajes desagradables o hirientes en los últimos doce meses, especificando que el $18 \%$ señaló que publicaron ese tipo de mensajes en plataformas donde otros podían verlos, el 14 $\%$ reportó que recibió amenazas por Internet y el $11 \%$ manifestó que quedó excluido de un grupo o actividad en línea (UNICEF, 2016).

En los últimos años, en nuestro país, los términos bullying y cyberbullying han estado presentes en los ámbitos profesionales -en publicaciones y eventos científicos- así como en la población general -en charlas a la comunidad, campañas gráficas y medios de comunicación-. Los medios de comunicación, entre ellos la prensa escrita, constituyen una interesante fuente de conocimiento ya que reflejan aquella información que se difunde entre la población y que alcanza a un segmento extenso de personas (Sarudiansky, 2016). Sin embargo, en el discurso mediático resulta esperable encontrar ciertas características específicas y diferenciales que lo distinguen de otros discursos, y que influyen en la comprensión, actitudes y juicios de valor del público general sobre un tema determinado (Dimopoulos y Koulaidis, 2002; Nelkin, 1995). En este sentido, la prensa escrita desarrolla un rol importante en la construcción pública de la experiencia común y del sentido colectivo ya que disemina información, concede legitimidad o significación pública a un evento, actor o fenómeno ofreciendo representaciones selectivas de ellos (Schudson, 2003). Siguiendo esta línea, y teniendo en cuenta que la lectura y análisis de aquello que publica la prensa respecto de un fenómeno permitiría observar aspectos interesantes del proceso de difusión de la información, del discurso empleado y de la significación pública que le otorga (Freidin y Ballesteros, 2012), el objetivo general de este trabajo se orienta a indagar el tipo de información y analizar su contenido respecto del fenómeno de $\mathrm{CB}$ en la prensa escrita argentina.

\section{Método}

Teniendo en cuenta el objetivo general del trabajo, el presente estudio es de tipo explo- 
ratorio y utiliza una metodología de enfoque cualitativo en lo atinente a los procedimientos de recolección y análisis de la información.

Se realizó una búsqueda de artículos periodísticos publicados en diferentes portales digitales de diarios editoriales argentinos. Dicha búsqueda fue realizada a partir de dos únicos descriptores: cyberbullying y ciberbullying teniendo en cuenta que, de acuerdo con lo hallado en la bibliografía sobre la temática, suelen utilizarse ambas expresiones para referir al fenómeno. La misma abarca el período comprendido entre el mes de enero del año 2006 hasta el mes de diciembre del año 2016.

Para el tratamiento de la información recolectada se realizó un análisis temático y de contenido considerando los aportes propuestos por Braun y Clarke (2006) -vinculados específicamente con el análisis temático- a fin de realizar una codificación de la información hallada que permita organizar el material, y obtener una descripción profunda y detallada del fenómeno.

En una primera etapa se realizó la lectura de cada uno de los artículos periodísticos y se utilizaron categorías propias y generales a fin de obtener un primer ordenamiento de la información recolectada. Dichas categorías incluyeron: fecha de publicación de los artículos, nombre del medio/portal digital y de la sección en los que fueron publicados, descriptor que se asoció con la búsqueda $\mathrm{y}$ código numérico de identificación.

Luego, se realizó la relectura de cada uno de los artículos periodísticos y se procedió a la simplificación y selección de la información mediante la identificación de distintos elementos significativos referidos a fragmentos del material (reducción de los datos). Posteriormente, se realizó una codificación abierta de la información mediante un proceso de comparación constante (generación de códigos iniciales) para luego organizar los datos en temas o categorías de análisis. De esta manera, se generaron diez temas de análisis que se presentan en la sección de Resultados.

Posteriormente, se realizó el análisis cualitativo del contendido propiamente dicho, el cual fue acompañado por un análisis cuantitativo que solo incluyó el cálculo de frecuencias porcentuales a fin de esbozar con mayor claridad los resultados y extraer conclusiones.

La información recolectada fue ordenada en una matriz para facilitar su sistematización y análisis.

\section{Resultados}

\section{La difusión y el tipo de información brindada por la prensa escrita argentina respecto del fenómeno de CB}

En una etapa inicial, se hallaron 168 artículos periodísticos que se asociaron con los descriptores utilizados para realizar la búsqueda. Se descartaron aquellos artículos cuya información se repetía exactamente en otros -es decir, artículos que replicaban el mismo contenido citando la fuente principal-. De esta manera, se obtuvo una muestra total conformada por 131 artículos periodísticos publicados en nueve portales digitales de diarios editoriales argentinos: La Nación, Clarín, Télam, Diario Popular, Infobae, Diario Crónica, Infonews, Tiempo Argentino y Página/12. Setenta y tres $(55.7 \%)$ de ellos se asociaron con el descriptor cyberbullying, mientras que $58(44.3 \%)$ lo hicieron con el descriptor ciberbullying. En la Tabla 1 puede observarse en detalle la cantidad de artículos periodísticos que se refieren al fenómeno de $\mathrm{CB}$, dividida según los portales digitales de los diarios y los años en los que fueron publicados. 
Tabla 1

Cantidad de artículos periodísticos que se refieren al fenómeno de CB, dividida según el portal digital del diario y el año de publicación.

\begin{tabular}{|c|c|c|c|c|c|c|c|c|c|c|c|c|}
\hline & 2006 & 2007 & 2008 & 2009 & 2010 & 2011 & 2012 & 2013 & 2014 & 2015 & 2016 & Total \\
\hline La Nación & 1 & 4 & 5 & 1 & 4 & 2 & 4 & 2 & 7 & 3 & 4 & 37 \\
\hline Clarín & - & - & 1 & - & 2 & - & 1 & 5 & 4 & 12 & 10 & 35 \\
\hline Télam & - & - & - & - & - & - & - & - & 3 & 4 & 8 & 15 \\
\hline D. Popular & - & - & - & - & - & - & 1 & 2 & 1 & 4 & 3 & 11 \\
\hline Infobae & - & - & - & - & - & - & - & 2 & 2 & 3 & 3 & 10 \\
\hline D. Crónica & - & - & - & - & - & - & - & 1 & 4 & 2 & 2 & 9 \\
\hline Infonews & - & - & - & - & - & 1 & 3 & 1 & 1 & - & - & 6 \\
\hline T. Argentino & - & - & - & - & - & - & 1 & - & 4 & - & - & 5 \\
\hline Página/12 & 1 & - & - & - & - & - & - & - & 2 & - & - & 3 \\
\hline Total & 2 & 4 & 6 & 1 & 6 & 3 & 10 & 13 & 28 & 28 & 30 & 131 \\
\hline
\end{tabular}

Teniendo en cuenta los años de publicación, se observa poca fluctuación en la cantidad de artículos publicados hasta el año 2011 al tiempo que se registra una tendencia creciente del número de publicaciones a partir del año 2012 -pudiendo observarse que más de tres cuartos de los artículos relevados (109 [83.2\%]) corresponden a los años 2012 en adelante-. A su vez, se observa una segunda tendencia creciente en el año 2014 a partir del cual se ubica el $47.5 \%$ (86) de los artículos considerados en este estudio (Figura 1).

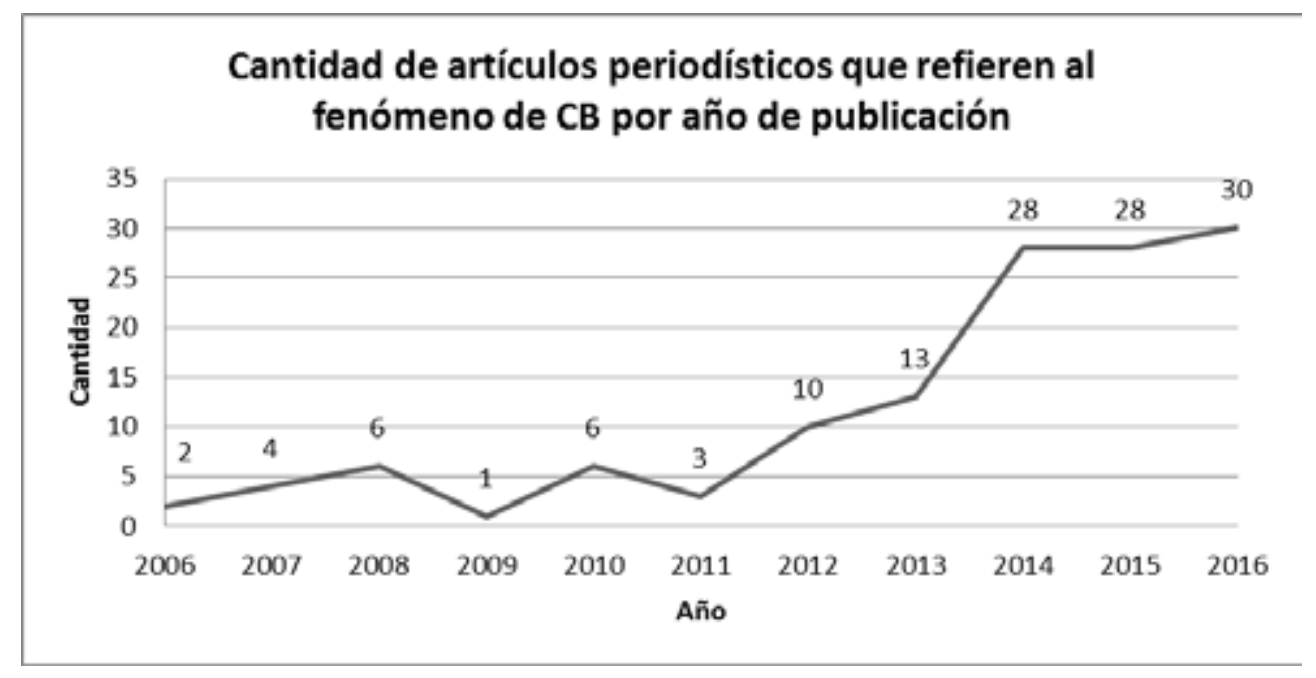

Figura 1. Cantidad de artículos periodísticos que se refieren al fenómeno deCB por año de publicación $(\mathrm{N}=131)$.

En lo que se refiere a las secciones de los portales digitales en las que fueron publicados los artículos, puede observarse una gran variabilidad: el fenómeno de CB ha sido abordado mayormente en las secciones denominadas
"Sociedad", "Interés general" y "Tecnología", $\mathrm{y}$ en menor medida en las secciones denominadas "Educación", "Comunidad" y "Ciencia y Salud” (Figura 2). 


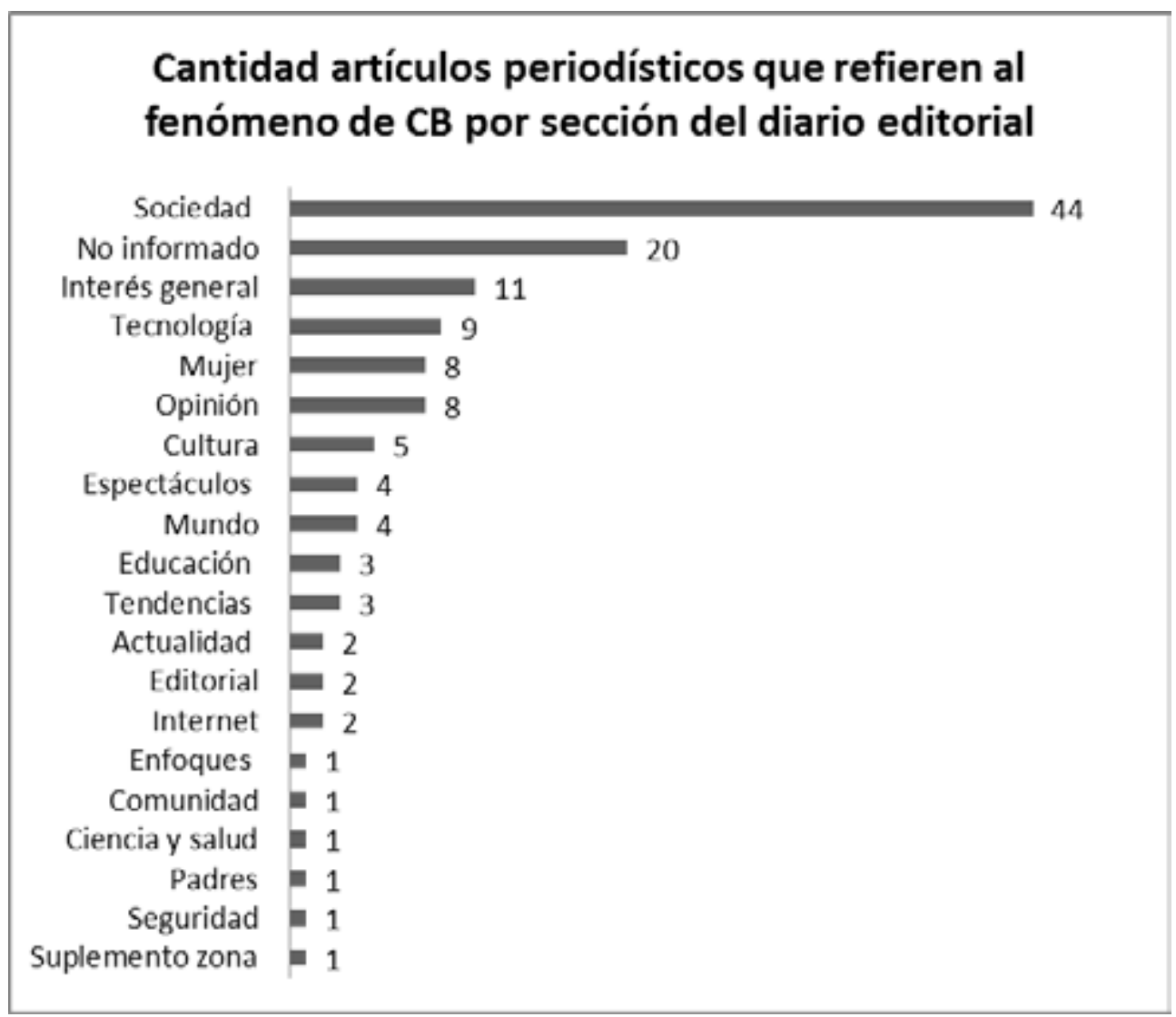

Figura 2. Cantidad de artículos periodísticos que se refieren al fenómeno de CB por sección de publicación del portal digital del diario $(\mathrm{N}=131)$.

Por otro lado, considerando la totalidad de los artículos seleccionados para este análisis, aproximadamente un tercio utiliza otras denominaciones -posibles traducciones lingüísticas- para referirse al fenómeno de CB: "ciberacoso" (13), "acoso cibernético" (11), "acoso virtual" (10), "cyberacoso" (3), "bullying virtual" (2), "acoso a través de Internet" (2), "intimidación cibernética" (1), "acoso digital" (1), "intimidación online" (1), "ciber intimidación" (1) e "intimidación virtual" (1).

En cuanto al tipo de información brindada en los artículos periodísticos, se observa que la misma refiere tanto a la definición y caracterización del fenómeno de CB como a las consecuencias, detección y posibles abordajes de esta problemática. Si bien el término "bullying" no fue incluido dentro de los descriptores que se utilizaron para realizar la búsqueda en los diferentes portales digitales de los diarios editoriales, se ha hallado que parte de los artículos relevados hacen referencia a este fenómeno brindando información relacionada con su definición, casos y testimonios, y posibles consecuencias. Así, teniendo en cuenta la variabilidad de la información brindada en los artículos, se generaron diez temas o categorías de análisis: 1. Definición de cyberbullying, 2. Definición de bullying, 3. Profesionales e instituciones especializadas, 4. Casos de bullying/cyberbullying, 5. Testimonios referidos a bullying/cyberbullying, 6. Modalidades de cyberbullying, 7. Indicadores para la detección del cyberbullying, 8. Consecuencias del cyberbullying, 9. Consecuencias del bullying, y 10. Abordajes posibles del fenómeno de cyberbullying (Tabla 2). 
Tabla 2

Tipo de información brindada en los artículos periodísticos.

\begin{tabular}{|c|c|}
\hline & $\mathrm{n}(\%)$ \\
\hline Definición de cyberbullying & $76(58.1)$ \\
\hline Definición de bullying & $29(22.1)$ \\
\hline Profesionales e instituciones especializadas & $89(67.9)$ \\
\hline Casos de bullying/cyberbullying & $38(29.1)$ \\
\hline $\begin{array}{c}\text { Testimonios referidos a bullying/ } \\
\text { cyberbullying }\end{array}$ & $14(10.7)$ \\
\hline Modalidades de cyberbullying & $48(36.6)$ \\
\hline $\begin{array}{c}\text { Indicadores para la detección del } \\
\text { cyberbullying }\end{array}$ & $11(8.4)$ \\
\hline Consecuencias del cyberbullying & $25(19.1)$ \\
\hline Consecuencias del bullying & $23(17.6)$ \\
\hline Abordajes posibles del cyberbullying & $80(61.1)$ \\
\hline
\end{tabular}

\section{El contenido de la información brindada por la prensa escrita argentina respecto del fenómeno de $\mathrm{CB}$}

A continuación se presentan los hallazgos obtenidos a partir del análisis de contenido correspondiente a cada uno de los temas de análisis.

\section{Definición de cyberbullying}

Este tema de análisis reúne aquellas definiciones del fenómeno de $\mathrm{CB}$ brindadas en los artículos periodísticos incluidos en este estudio. De los 76 artículos que brindan una definición sobre el fenómeno, 21 (27.6 \%) mencionan alguna fuente proveniente, principalmente, de la consulta a especialistas en la temática. En cuanto a las definiciones ofrecidas, la mayoría de los artículos periodísticos (48 [63.1 \%]) definen el CB como el acoso $\mathrm{u}$ hostigamiento que tiene lugar a través de la utilización de herramientas tecnológicas de comunicación haciendo referencia a redes sociales, aplicaciones de mensajería instantánea para teléfonos inteligentes, correo electrónico, blogs y otros sitios de la web. A su vez, en el $26.3 \%$ (20) de los artículos periodísticos se menciona que se trata de un acoso que sucede entre pares, mientras que en el $21 \%$ (16) se señala que el CB constituye la continuidad del bullying o maltrato escolar presencial que sucede entre los/as alumnos/ as dentro de la escuela. En menor medida, se explicita en los artículos que el $\mathrm{CB}$ incluye humillaciones y burlas a través de internet (14 [18.4\%]), que es un fenómeno que afecta a niños/as y adolescentes (13 [17.1\%]), que se trata de un comportamiento repetitivo y prolongado en el tiempo (10 [13.2\%]), y que puede incluir comportamientos amenazantes o intimidatorios en línea (8 [10.5\%]). La masividad y alcance, la falta de temporalidad debida a la instantaneidad que posibilitan las tecnologías de la información y la comunicación, la intencionalidad y la posibilidad de anonimato digital son mencionadas como características del $\mathrm{CB}$ en el $10.5 \%, 7.9 \%$, $5.3 \%$ y $5.3 \%$ de los artículos periodísticos $(8$, 6,4 y 4), respectivamente. El hecho de que el CB pueda ser llevado a cabo de manera individual o grupal, la existencia de un desequilibrio de poder y que, a diferencia del fenómeno de grooming, no involucra el engaño de un adulto hacia un menor con un fin de abuso sexual son mencionados, en igual medida, en el $4 \%$ (3) de los artículos. 


\section{Definición de bullying}

Este tema de análisis reúne a aquellas definiciones del fenómeno de bullying brindadas en los artículos periodísticos incluidos en este estudio. De los 29 artículos que brindan una definición sobre el fenómeno de bullying, 13 (44.8\%) mencionan alguna fuente de la misma proveniente, principalmente, de la consulta a especialistas en la temática. En cuanto a las definiciones brindadas, el $58.5 \%$ (17) de los artículos menciona que el bullying tiene lugar entre pares, mientras que el $51.7 \%$ (15) refiere que se trata de un comportamiento repetitivo y prolongado en el tiempo. El $41.4 \%$ (12) señala que se trata de un maltrato físico y verbal, mientras que el $31 \%$ (9) menciona que implica conductas de acoso y hostigamiento. La existencia de un desequilibrio de poder y la intencionalidad son atribuidas como características del bullying en igual medida por el $20.7 \%$ (6) de los artículos. En proporciones algo menores, se observan menciones relacionadas con que el bullying puede ser llevado a cabo de manera individual o grupal (4 [13.8\%]), que incluye conductas relacionadas con la exclusión o aislamiento social $(13.8 \%)$, con la intimidación o amenaza (3 [10.4\%]) y con burlas y humillaciones (10.4 \%). El $6.9 \%$ (2) menciona el tipo de bullying sexual y, en igual proporción, se señala que se trata de un fenómeno social complejo.

\section{Profesionales e instituciones especializadas}

Este tema de análisis reúne a aquellos profesionales e instituciones que son consultados y/o mencionados en los artículos periodísticos incluidos en este estudio. En lo que refiere a los profesionales consultados se destaca que $24.7 \%$ (22) de los artículos mencionan a Licenciados en Psicología, $13.5 \%$ (12) a Licenciados en Ciencias de la Educación, $12.4 \%$ (11) a investigadores, $8.9 \%(8)$ a especialistas en tecnologías y seguridad informática, 5.7\% (5) a Licenciados en Ciencias de la Comunicación y 3.4 \% (3) a abogados. En cuanto a las instituciones especializadas, se observa la referencia de algunos organismos tanto nacionales como internacionales (por ejemplo, Equipo Antibullying Argentina, Equipo Bullying Cero Argentina, Libres de Bullying, Organización No Gubernamental [ONG] Bullying sin Fronteras, ONG Argentina Cibersegura, Observatorio de Convivencia Escolar de la Universidad Católica Argentina, Programa Con vos en la Web -dependiente del Ministerio de Justicia y Derechos Humanos de la Nación Argentina-, Foro Generaciones Interactivas, www.segu-kids.org). Asimismo, y en menor medida, se observa la referencia a organismos más amplios que abordarían las problemáticas de bullying y $\mathrm{CB}$ entre otras temáticas (por ejemplo, Consejo de los Derechos de Niñas, Niños y Adolescentes de la Ciudad de Buenos Aires, Argentina; Fiscalía de Delitos Informáticos de la Ciudad de Buenos Aires, Argentina; Defensoría del Pueblo de la Ciudad de Buenos Aires, Argentina; Instituto Nacional Contra la Discriminación, la Xenofobia y el Racismo, Argentina; Ministerio de Educación de la Nación Argentina; Organización Mundial de la Salud [OMS]; Fondo de las Naciones Unidas para la Infancia [UNICEF]).

\section{Casos de bullying/cyberbullying}

Este tema de análisis reúne a aquellos casos que son mencionados en los artículos periodísticos incluidos en este estudio. De los 38 artículos que mencionan casos de maltrato escolar, 24 (63.2 \%) hacen referencia a casos locales (15 casos de bullying y nueve casos de $\mathrm{CB}$ ) entre los que se mencionan cuatro casos de suicidio y un caso de homicidio. Los 14 artículos restantes $(36.8 \%)$ hacen referencia a casos internacionales, que incluyen 11 casos de suicidio y un caso de homicidio ocurridos en distintos países (Estados Unidos, Canadá, Alemania, Gran Bretaña e Italia). 


\section{Testimonios referidos a bullying/ cyberbullying}

Este tema de análisis reúne a aquellos testimonios que son mencionados en los artículos periodísticos incluidos en este estudio. De los 14 artículos que mencionan algún tipo de testimonio, siete $(50 \%)$ refieren testimonios brindados por algún familiar de un niño, niña o adolescente victimizado, tanto mediante tecnologías de comunicación electrónica (5) como presencialmente (2); cuatro (28.6\%) refieren testimonios de docentes con respecto al fenómeno de bullying (1) y CB (3) y tres $(21,4 \%)$ reflejan el testimonio de adolescentes relacionados con la problemática del CB.

\section{Modalidades de cyberbullying}

Este tema de análisis reúne a aquellas modalidades o formas de ejercer el CB que son mencionadas en los artículos periodísticos incluidos en este estudio. Al respecto, la difusión de imágenes y/o videos en la web, los comentarios crueles, despectivos y de burla, junto con el envío de mensajes ofensivos y amenazantes, han sido las modalidades mayormente mencionadas en los artículos periodísticos (13 [27.1 \%], 12 [25\%] y 11 [22.9\%], respectivamente). A su vez, el 18.8\% (9) menciona la creación de grupos en línea para agredir a una persona. La difusión de rumores y/o contenidos difamatorios junto con la suplantación o robo de identidad en línea han sido mencionadas en igual proporción (7 [14.6\%]). Asimismo, y en igual medida (6 [12.5\%]), se señala la difusión de información privada y la creación de perfiles falsos. El $10.4 \%$ (5) de los artículos menciona la difusión de fotografías alteradas o trucadas, mientras que el $6.3 \%$ (3) señala el robo de contraseñas y la exclusión de grupos en línea como otras modalidades en que el CB puede ser llevado a cabo. En menor medida se mencionan la creación de memes (2 [4.2\%]), el envío de spam (1 [2.1\%]), las falsas acusaciones de pedofilia y delincuencia $(2.1 \%)$, el envío de falsos mensajes suicidas en nombre de una persona
(2.1\%), la creación de videos musicales con el fin de humillar $(2.1 \%)$, la creación de eventos falsos $(2.1 \%)$ y la inducción al suicidio mediante el envío de mensajes y comentarios $(2.1 \%)$.

\section{Indicadores para la detección del cyberbullying}

Este tema de análisis reúne a aquellos indicadores para la detección del CB que son mencionados en los artículos periodísticos incluidos en este estudio. Al respecto, la mayoría de los artículos señala indicadores relacionados con posibles alteraciones del estado de ánimo y del comportamiento de la persona que pudiera estar padeciendo situaciones de CB. Así, el 54.5 \% (6) de los artículos menciona el temor y la reticencia para ir a la escuela y la presencia de síntomas de ansiedad. El bajo rendimiento escolar, los cambios en el ritmo del sueño, insomnio y pesadillas, y la presencia de síntomas físicos -como los dolores de estómago y de cabezason mencionados en igual medida por el $45.5 \%$ (5) de los artículos. A su vez, el aislamiento social, la irritabilidad y los sentimientos de vergüenza y autoculpabilización son señalados en igual proporción (4 [36.4\%]). Otros indicadores mencionados son la existencia de un bajo nivel de autoestima (3 [27.3\%]) y en igual proporción (2 [18.2\%]), la presencia de sintomatología depresiva, la frecuente pérdida o rotura de objetos personales, la evitación de recreos o actividades escolares y los trastornos de la conducta alimentaria. En relación con posibles indicadores referidos a la persona que ejercería el $\mathrm{CB}$, se destaca que solo cuatro artículos hacen referencia al respecto, mencionando la presencia de un nivel de autoestima elevado, la capacidad de poder y liderazgo y la falla en la regulación de los impulsos.

\section{Consecuencias del cyberbullying}

Este tema de análisis reúne las consecuencias posibles que el fenómeno de $\mathrm{CB}$ 
puede ocasionar, mencionadas en los artículos periodísticos incluidos en este estudio. La mayoría de los artículos (11 [44 \%]) menciona la concreción del suicidio como la principal consecuencia del CB. La presencia de síntomas y/o trastornos depresivos es señalada por el $24 \%$ (6) de los artículos, mientras que los intentos de suicidio y la afectación de la autovaloración son mencionadas por el $16 \%$ (4). También se menciona el homicidio, y la presencia de síntomas y/o trastornos de ansiedad (3 [12\%]), el abuso de sustancias, el aislamiento social, la presencia de sentimientos de desamparo e indefensión y las alteraciones del sueño (2 [8\%]) y en menor medida (1 [4\%]), las lesiones físicas graves y el traslado de conductas antisociales y psicopáticas a otras relaciones interpersonales (familiares, amorosas, etc.).

\section{Consecuencias del bullying}

Este tema de análisis reúne las consecuencias posibles que el bullying puede ocasionar, mencionadas en los artículos periodísticos incluidos en este estudio. La mayoría de los artículos (10 [43.5\%]) menciona la concreción del suicidio como la principal consecuencia del bullying. En menor medida se mencionan los intentos de suicidio y la afectación de la autovaloración (5 [21,74\%]), el ausentismo escolar, el homicidio y la presencia de síntomas y/o trastornos depresivos (4 [17.4 \%]), las lesiones físicas graves, la presencia de síntomas psicosomáticos, la existencia de síntomas y/o trastornos de ansiedad y la afectación de las relaciones interpersonales (3 [13\%]). El consumo de sustancias y el aislamiento social son mencionados por el $8.7 \%$ (2) de los artículos, mientras que, en igual proporción (1 [4.3\%]), se señalan los trastornos de la conducta alimentaria, la presencia de síntomas del trastorno de estrés postraumático, el bloqueo o embotamiento afectivo, la presencia de sentimientos de desamparo e indefensión y la fuga del hogar.

\section{Abordajes posibles del cyberbullying}

Este tema de análisis reúne los posibles abordajes del fenómeno de $\mathrm{CB}$ que son mencionados en los artículos periodísticos incluidos en este estudio. El acompañamiento de los padres en las actividades en línea de sus hijos e hijas - incluyendo el diálogo fluido entre padres e hijos/as, la conversación sobre situaciones incómodas vividas en la web y el establecimiento de reglas de uso de Internetse observa como una de las principales estrategias de abordaje señalada por el $28.8 \%$ (23) de los artículos periodísticos. El establecimiento de reglas de conductas aceptables e inaceptables, tanto en línea como fuera de línea, mencionando las expresiones de "ciudadanía digital" y "ciber-conducta", fueron señaladas por el $19 \%$ (15) de los artículos como otra de las posibles estrategias de abordaje. A su vez, el desarrollo de la sensibilidad y de la capacidad empática junto con el ejercicio de conductas de reparación del daño fueron mencionadas por el $17.5 \%$ (14). El hecho de brindar información a menores, docentes y padres acerca de herramientas de seguridad, privacidad y administración de perfiles en línea fue señalado por el $16.3 \%$ (13). Otras estrategias observadas se relacionan con la supervisión y el control de los padres respecto de las actividades que sus hijos/as realizan en línea (12 [15 \%]), con el hecho de brindar a docentes información y capacitación acerca del fenómeno (11 [13.8\%]), con las intervenciones preventivas desde la escuela -por ejemplo, estableciendo acuerdos de convivencia- (8 [10\%]), con el abordaje de la problemática dentro de las aulas (7 [8.8\%]) y con el trabajo con todo el grupo de pares $(8.8 \%)$. En menor medida se observan posibles estrategias de abordaje relacionadas con el hecho de no naturalizar el fenómeno (6 [7.5\%]), educar en el respeto hacia sí mismo y hacia los demás (4 [5\%]), la consideración de variables contextuales - escolares, familiares, sociales- $(5 \%)$, el establecimiento de políticas públicas $(3[3.8 \%])$ y la concientización social $(3.8 \%)$. 


\section{Discusión}

Este estudio tenía como objetivo indagar el tipo de información y analizar el contenido de la misma respecto del fenómeno de $\mathrm{CB}$ en la prensa escrita argentina. Respecto de la difusión, se observa que la atención que la prensa brinda a este fenómeno presenta una tendencia creciente durante los últimos años. En lo que respecta al incremento de la cantidad de artículos periodísticos publicados a partir del año 2012 podría inferirse cierta relación entre tal aumento y el caso de la estudiante canadiense de 15 años llamada Amanda Todd, quien cuatro horas antes de concretar su suicidio compartió en una red social un video en el cual relataba sus experiencias de maltrato escolar y su sufrimiento por ser agredida tanto física como emocionalmente, de manera presencial y mediante tecnologías de comunicación electrónica (Noronha, 2012). Dicho video se convirtió en un "fenómeno viral" y fue noticia en muchos medios de comunicación de distintos países (Wong-Lo y Bullock, 2014). En relación con el segundo incremento en la cantidad de publicaciones registrado a partir del año 2014, cabe considerar la aprobación de la Ley Nacional para la Promoción de la Convivencia y el Abordaje de la Conflictividad Social en las Instituciones Educativas, sancionada un año antes en Argentina. En relación con el fenómeno de $\mathrm{CB}$, si bien en la mencionada ley no se utiliza esta denominación, se observa que en su artículo 3, inciso $\mathrm{c}$, menciona que uno de sus principios orientadores es "el respeto y la aceptación de las diferencias, el rechazo a toda forma de discriminación, hostigamiento, violencia y exclusión en las interacciones entre los integrantes de la comunidad educativa incluyendo las que se produzcan mediante entornos virtuales y otras tecnologías de la información y comunicación" (Ley No 26.892, 2013). Asimismo, en el año 2013, se sancionó la Ley $\mathrm{N}^{\circ} 26.904$, la cual aprueba una modificación al Código Penal argentino incorporando al artículo 131 que "será penado con prisión de seis meses a cuatro años el que, por medio de comunicaciones electrónicas, telecomunicaciones o cualquier otra tecnología de transmisión de datos, contactare a una persona menor de edad, con el propósito de cometer cualquier delito contra la integridad sexual de la misma" (Ley $\mathrm{N}^{\circ} 26.904,2013$ ). Esta última ley fue llamada informalmente "ley de grooming", aunque es necesario destacar que el grooming refiere a una problemática diferenciada del CB (Montiel, Carbonell y Salomm, 2014; Wachs, Jiskrova, Vazsonyi, Wolf y Junger, 2016) y que constituye, a diferencia de este último, un delito penal que debe ser denunciado en nuestro país. En este sentido, se podría inferir que la prensa escrita otorgó más atención a estos fenómenos, incluido el $\mathrm{CB}$, conforme a los avances institucionales que se fueron desarrollando en los últimos años en Argentina.

Otro aspecto a destacar respecto de la difusión del fenómeno del $\mathrm{CB}$ gira en torno a las distintas denominaciones utilizadas para mencionarlo ("ciberacoso", "acoso cibernético", "acoso virtual", etc.) las cuales darían cuenta de una falta de consenso en lo que refiere a la traducción al idioma español para referirse a dicho fenómeno en la prensa escrita argentina.

Con respecto al tipo de información, se observa que la prensa escrita brinda en mayor medida información relacionada con las características y abordajes posibles del $\mathrm{CB}$, $\mathrm{y}$ en menor medida con su detección, aspecto a tener en cuenta considerando las amplias repercusiones negativas que este fenómeno puede generar.

En cuanto al contenido de la información, el análisis realizado en este estudio permite observar que, en lo que refiere a las definiciones del fenómeno de CB brindadas por la prensa escrita, se destaca la baja proporción de artículos en los que son mencionadas las tres características básicas del CB referidas a la intencionalidad, la repetición en el tiempo y la existencia de un desequilibrio de poder. En este sentido, se deben tener en cuenta las particularidades que estas características adquieren 
considerando el medio tecnológico por el cual se desarrolla el maltrato -aspecto que ha sido abordado en la introducción de este trabajo-y que podría explicar la baja proporción de artículos en los que son mencionadas. También, cabe destacar que el hecho de que la prensa brinde escasa información acerca de la existencia de un desequilibrio de poder constituye un aspecto importante, ya que la consideración u omisión de esta característica básica del CB repercutiría en las estrategias empleadas para su abordaje por parte de la población general; por ejemplo, en el empleo de mediaciones o negociaciones las cuales redundarían en una revictimización (Ministerio de Educación Pública de Costa Rica/UNICEF, 2015).

Por otro lado, las modalidades de CB referidas por la prensa escrita expresan una gran variabilidad de formas en las que este fenómeno puede ser llevado a cabo, lo cual coincide con lo reportado en investigaciones sobre la temática (e.g. Bauman, 2015; Kowalski et al., 2012; Willard, 2005, 2007). Pero se debe tener en cuenta que nuevas formas de agresión podrían tener lugar en el futuro -considerando la velocidad del avance tecnológico y la multiplicidad de herramientas y recursos utilizados eventualmente por los estudiantes para agredir a sus pares- cuestión que requiere de una constante actualización en este campo al momento de informar acerca de las modalidades que puede adoptar el CB.

Otro punto de interés refiere a los profesionales consultados por la prensa escrita, donde se destaca que se recurre principalmente a licenciados en Psicología, y en menor medida a otros profesionales, para que brinden sus conocimientos y perspectivas acerca del fenómeno de CB. Cabe la pregunta acerca de si la prensa considera que esta problemática se relaciona con el campo de la salud, ya sea considerando sus posibles causas como sus consecuencias. En relación con lo anterior, algunos investigadores consideran al $\mathrm{CB}$ como un problema emergente de salud pública (Bickham y Rich, 2009; David-Ferdon y Feldman-Hertz, 2007). Al respecto, resulta llamativo que la gran mayoría de los artículos periodísticos relevados en este estudio no fueran publicados en las secciones vinculadas con la salud de los portales digitales de los diarios. Asimismo, cabe mencionar que las consecuencias del fenómeno de CB principalmente reflejadas por la prensa refieren a alteraciones emocionales y de comportamiento, aspecto que puede vincularse con el hecho de que la mayoría de los profesionales a quienes la prensa consulta sean licenciados en Psicología.

En lo que refiere a los casos de CB mencionados por la prensa, se observan tanto de procedencia local como internacional. Al respecto, un aspecto a destacar es que los casos con mayor repercusión mediática son aquellos en donde se produce la muerte por suicidio del niño, niña o adolescente presentándola como una consecuencia directa del bullying o CB. En este sentido, vale mencionar que si bien la agresión y victimización son problemas críticos en la vida de muchos niños, niñas y adolescentes, el intento y/o concreción del suicidio no es un hecho aislado sino que suele estar asociado a otros factores, como los trastornos de personalidad, algunos trastornos mentales, el abuso de sustancias, los conflictos en las relaciones interpersonales, entre otros (Orpinas y Horne, 2015). Siguiendo esta línea, no se trataría entonces de una relación causal directa sino que podría ser un elemento más en el entramado complejo del suicidio.

En cuanto a las instituciones mencionadas, además de las especializadas en bullying y $\mathrm{CB}$, se mencionan otras vinculadas con los derechos de niños, niñas y adolescentes (por ejemplo, el Consejo de los Derechos de Niñas, Niños y Adolescentes de la ciudad, y UNICEF), otras relacionadas con el campo judicial (por ejemplo, la Fiscalía de Delitos Informáticos de la ciudad) y otras relacionadas con la salud (por ejemplo, la OMS). De allí que se pueda inferir que la prensa intentaría reflejar la complejidad del fenómeno, los distintos niveles involucrados y el posible abordaje multidisciplinar que este requeriría, 
cuestión que parecería entrar en contradicción con el registro de cierto "sesgo hacia la individualización". Es decir, en este sentido, y en cuanto a los indicadores para la detección del $\mathrm{CB}$, si bien son escasos los artículos que hacen mención a ellos, se observa que la mayoría se vincula con las consecuencias negativas de esta problemática en la salud psicológica de los implicados, destacándose indicadores y consecuencias individuales, sin hacer referencia a indicadores y consecuencias grupales, institucionales y/o sociales. El desarrollo de un clima escolar hostil e insolidario, la normalización de las conductas agresivas, la valoración positiva de los comportamientos violentos a fin de obtener éxito social, y posibles consecuencias en el profesorado y a nivel familiar no son mencionadas por la prensa. Este sesgo hacia la individualización de las consecuencias también se vería reflejado en los distintos abordajes posibles que refieren los artículos. En este sentido, el trabajo con todo el grupo de pares, la consideración de variables contextuales, el establecimiento de políticas públicas y la concientización social son los abordajes menos señalados, es decir, aquellos que favorecerían el desarrollo de abordajes integrales y ecológicos considerando las características del contexto psicosocial más amplio en el cual este fenómeno se produce. Cabra-Torres y Marciales-Vivas (2012) sostienen que uno de los mitos del CB se relaciona con la creencia según la cual controlando la tecnología y a los/as jóvenes se obtendrá como resultado el control de las expresiones de acoso y maltrato, señalando que lo que debe priorizarse al momento de reflexionar sobre esta temática es que el CB constituye un problema social y no un problema de las tecnologías. En este sentido, solo dos artículos mencionan que el bullying se trataría de un fenómeno social complejo mientras que no se hallaron referencias de este tipo en lo que refiere al CB. Este último punto abre la pregunta acerca de cómo la prensa presenta al fenómeno de $\mathrm{CB}$, si transmite información acerca de sus múltiples aristas y complejidades y si lo considera un fenómeno vinculado con variables más allá de las individuales, destacando que la sección en la cual se publican principalmente los artículos periodísticos sobre esta problemática es la que refiere a "Sociedad".

Por último, cabe resaltar la baja proporción de artículos en los que se mencionan testimonios relacionados con esta problemática, sobre todo en lo que refiere a los testimonios brindados por los propios adolescentes -con el debido resguardo y protección de su identidad- . Este aspecto ha de ser destacado ya que reflejaría que la prensa escrita le otorga poco lugar al punto de vista de los alumnos/as quienes, desde sus vivencias, actitudes y perspectivas sobre el fenómeno que los atraviesa, podrían realizar aportes importantes tanto para la reflexión de estrategias de prevención como para el desarrollo de herramientas de abordaje efectivas.

Para finalizar, es necesario mencionar algunas limitaciones del presente estudio. En este sentido, cabe aclarar que los diferentes diarios editoriales iniciaron la digitalización de sus ejemplares en distintos años, lo cual podría incidir en la cantidad total de artículos relevados y en la cantidad registrada para cada uno de los portales digitales. Asimismo, para este estudio se utilizaron solo dos descriptores para realizar la búsqueda por lo que futuros trabajos podrían incorporar otros términos a fin de ampliar y profundizar los hallazgos. Lo anterior también podría contribuir a generar otras categorías de análisis de manera tal de registrar otros aspectos relacionados con el tipo y contenido de la información no abordados en este estudio. Aún con estas limitaciones, lo hallado mediante el análisis de los artículos relevados en este trabajo permite observar algunos aspectos de interés acerca de la manera en que se difunde la información sobre el CB en la población general a través de la prensa escrita argentina. Como fue señalado, este aspecto es de relevancia dado el impacto que tienen los medios de comunicación en las creencias y actitudes de las personas, en las maneras de representar y significar los fenó- 
menos y en los modos de intervenir en ellos. En este sentido, este estudio podría contribuir a la ampliación del conocimiento sobre el CB en nuestra región y eventualmente, al desarrollo de intervenciones efectivas para favorecer el adecuado abordaje de esta problemática.

\section{Referencias bibliográficas}

Aboujaoude, E., Savage, M.W., Starcevic, V. y Salame, W.O. (2015). Cyberbullying: Review of an Old Problem Gone Viral. Journal of Adolescent Health, 57(1), 10-18. http://dx.doi. org/10.1016/j.jadohealth.2015.04.011

Ayestarán-Etxeberria, S. (1987). El grupo de pares y el desarrollo psicosocial del adolescente. Estudi General: Revista de la Facultat de Lletres de la Universitat de Girona, 7, 123-136.

Bauman, S. (2015). Types of Cyberbullying. En S. Bauman (Ed.), Cyberbullying (pp. 53-58). Alexandria, VA, USA: American Counseling Association. http://dx.doi. org/10.1002/9781119221685.ch4

Bickham, D.S. y Rich, M. (2009). Global assessment of online threats and intervention opportunities for adolescents. Journal of Adolescent Health, 44, 24-25. http://dx.doi.org/10.1016/j. jadohealth.2008.10.053

Braun, V. y Clarke, V. (2006). Using thematic analysis in psychology. Qualitative Research in Psychology, 3(2), 77-101. http://dx.doi. org/10.1191/1478088706qp063oa

Cabra-Torres, F. y Marciales-Vivas, G.P. (2012). Comunicación electrónica y cyberbullying: Temas emergentes para la investigación e intervención socioeducativa. Psicología desde el Caribe, 29(3), 707-730.

Carozzo, J., Benites, L., Zapata, L. y Horna, V. (2012). El bullying no es un juego. Guía para todos. Lima: Publicación del Observatorio Sobre Violencia y Convivencia en la Escuela. Recuperado de http://bp000695.ferozo.com/ wp-content/uploads/2012/11/Guia-de-Bullying-Observatorio.pdf

David-Ferdon, C. y Feldman-Hertz, M. (2007).
Electronic Media, Violence, and Adolescents: An Emerging Public Health Problem. Journal of Adolescent Health, 41(6), 1-5. http://dx.doi. org/10.1016/j.jadohealth.2007.08.020

Dehue, F., Bolman, C.y Vollink, T. (2008). Cyberbullying: Youngsters' experiences and parental perception. CyberPsychology \& Behavior, 11(2), 217-223. http://dx.doi.org/10.1089/ cpb. 2007.0008

Dimopoulos, K. y Koulaidis, V. (2002). The socio-epistemic constitution of science and technology in the Greek press: an analysis of its presentation. Public Understanding of Science, 11(3), 225-241. http://dx.doi. org/10.1088/0963-6625/11/3/302

Dooley, J.J., Gradinger, P., Strohmeier, D., Cross, D. y Spiel, C. (2010). Cybervictimisation: The association between help-seeking behaviours and self-reported emotional symptoms in Australia and Austria. Australian Journal of Guidance \& Counselling, 20(2), 194-209. http://dx.doi.org/10.1375/ajgc.20.2.194

Dooley, J.J., Pyzalski, J. y Cross, D. (2009). Cyberbullying Versus Face-to-Face Bullying. A Theoretical and Conceptual Review. Zeitschriftür Psychologie / Journal of Psychology, 217(4), 182-188. http://dx.doi.org/10.1027/0044$\underline{3409.217 .4 .182}$

Erdur-Baker, Ö. y Tanrikulu, I. (2010). Psychological consequences of cyberbullying experiences among Turkish secondary school children. Procedia Social and Behavioral Sciences, 2(2), 2771-2776. http://dx.doi.org/10.1016/j. sbspro.2010.03.413

Fondo de las Naciones Unidas para la Infancia [UNICEF]. (2016). Kids Online/ Chic@s Conectados. Investigación sobre percepciones y hábitos de niños, niñas y adolescentes en internet $y$ redes sociales. Recuperado de http://www.unicef.org/argentina/spanish/ COM kidsonline2016.pdf

Freidin, B. y Ballesteros, M. (2012). La difusión transnacional de medicinas alternativas: la presencia del Ayurveda en la prensa argentina. Papeles de Trabajo, 6(10), 128-159.

Gámez-Guadix, M., Orue, I., Smith, P.K. y Calvete, 
E. (2013). Longitudinal and reciprocal relations of cyberbullying with depression, substance use, and problematic internet use among adolescents. Journal of Adolescent Health, 53, 446-452. http://dx.doi.org/10.1016/j.jadohealth.2013.03.030

Garalgordobil, M. (2014). Cyberbullying. Screening de acoso entre iguales: descripción y datos psicométricos. International Journal of Developmental and Educational Psychology. Revista INFAD de Psicología, 4(1), 311-318. http:// dx.doi.org/10.17060/ijodaep.2014.n1.v4.617

Grigg, D.W. (2010). Cyber-Aggression: Definition and Concept of Cyberbullying. Australian Journal of Guidance and Counselling, 20(2), 143-156. http://dx.doi.org/10.1375/ ajgc.20.2.143

Hinduja, S. y Patchin, J.W. (2010). Bullying, cyberbullying, and suicide. Archives of suicide research, 14(3), 206-221. http://dx.doi.org/10. $\underline{1080 / 13811118.2010 .494133}$

Juvoven, J. y Gross, E.F. (2008). Extending the school grounds? Bullying experiences in cyberspace. Journal of School Health, 78(9), 496-505. $\quad$ http://dx.doi.org/10.1111/j.17461561.2008.00335.x

Kowalski, R.M., Limber, S.P. y Agatston, P.W. (2012). Cyberbullying: Bullying in the Digital Age, 2nd Edition. Malden, MA: Wiley-Blackwell.

Kowalski, R. y Limber, S.P. (2013). Psychological, psysical, and academic correlates of cyberbullying and traditional bullying. Journal of Adolescen Health, 53(1), 13-20. http://dx.doi. org/10.1016/j.jadohealth.2012.09.018

Ley $\mathrm{N}^{\circ}$ 26.892, Boletín Oficial de la República Argentina, 11 de septiembre de 2013. Recuperado de https://www.boletinoficial.gob.ar/ web2/utils/pdfView?file=\%2Fpdf\%2Fnorma\%2F10344383\%2Fnull\%2FPrimera\%2FMuJB0gfXXQAq8BbJkIPIEi1bLS1JVilbLdFRcNC8qaZ36n0durjk8rE\%3D\%2F1

Ley $\mathrm{N}^{\circ}$ 26.904, Boletín Oficial de la República Argentina, 13 de noviembre de 2013. Recuperado de https://www.boletinoficial.gob.ar/ web2/utils/pdfView?file=\%2Fpdf\%2Fnorma\%2F10368912\%2Fnull\%2FPrimera\%-
2FslashBar1YaN6CujK0OMGB9c3bYRi1bLS1JVilbLZmC5A9kiHIdb3KsAfFYxzI\%3D\%2F1

Li, Q. (2006). Cyberbullying in Schools. School Psychology International, 27(2), 157-170. http://dx.doi.org/10.1177/0143034306064547

Mason, K.L. (2008). Cyberbullying: A preliminary assessment for school personnel. Psychology in the Schools, 45(4), 323-348. http://dx.doi. org/10.1002/pits.20301

Ministerio de Educación Pública de Costa Rica / Fondo de las Naciones Unidas para la Infancia [UNICEF]. (2015). Protocolo de actuación en situaciones de bullying. Recuperado de https:// www.unicef.org/costarica/Documento-Protocolo-Bullying.pdf

Monelos, M.E., Mendiri, P. y García-Fuentes, C.D. (2015). El bullying revisión teórica, instrumentos y programas de intervención. Revista de Estudios e Investigación en Psicología y Educación, 2, 74-78. http://dx.doi. org/10.17979/reipe.2015.0.02.1299

Montiel, I., Carbonell, E. y Salom, M. (2014). Victimización infantil sexual online: online grooming, ciber-abuso y ciber-acoso sexual. En M. Lameiras y E. Orts (Coords.), Delitos Sexuales contra menores. Abordaje psicológico jurídico y policial (pp. 203-224). Valencia: Tirant Lo Blanch.

Nelkin, D. (1995). Selling Science: How the Press Covers Science and Technology. New York: W.H. Freeman.

Noronha, C. (2012, 15 de octubre). Amanda Todd: Bullied teen shared story, then committed suicide. The Christian Science Monitor. Recuperado de https://www.csmonitor.com/ The-Culture/Family/2012/1015/Amanda-Todd-Bullied-teen-shared-story-then-committed-suicide

Olweus, D. (1998). Conductas de acoso y amenaza entre escolares. Madrid: Morata.

Olweus, D. y Limber, S.P. (2017). Some problems with cyberbullying research. Current Opinion in Psychology. 19, 139-143. http://dx.doi. org/10.1016/j.copsyc.2017.04.012

Oros, L.B. y Fontana Nalesso, A.C. (2015). Niños socialmente hábiles: ¿Cuánto influyen 
la empatía y las emociones positivas? Interdisciplinaria, Revista de Psicología y Ciencias Afines, 32(1), 109-125. http://dx.doi. org/10.16888/interd.2015.32.1.6

Orpinas, P. y Hornes, A.M. (2015). Suicidal ideation and bullying: an ecological examination of community impact. En P. Goldblum, D.L. Espelage, J. Chu y B. Bongar (Eds.), Youth Suicide and Bullying: Challenges and Strategies for Prevention and Intervention (pp. 50-63). New York: Oxford University Press.

Ortega-Barón, J., Torralba, E. y Buelga, S. (2017). Distrés psicológico en adolescentes víctimas de cyberbullying. Revista de Estudios e Investigación en Psicología y Educación, 4(1), 10-17. http://dx.doi.org/10.17979/ reipe.2017.4.1.1767

Ortega-Ruiz, R. (1994). Las malas relaciones interpersonales en la escuela: Estudio sobre la violencia y el maltrato entre compañeros de segunda etapa de E.G.B. Infancia y Sociedad, 27-28, 191-216.

Ortega-Ruiz, R. (2010). Treinta años de investigación y prevención del bullying y la violencia escolar. En R. Ortega-Ruiz (Ed.), Agresividad injustificada, bullying y violencia escolar (pp. 15-30). Madrid: Alianza Editorial.

Ortega-Ruiz, R. y Mora-Merchán, J.A. (2008). Las redes de iguales y el fenómeno del acoso escolar: Explorando el esquema dominio-sumisión. Infancia y Aprendizaje, 31(4), 515-528. http:// dx.doi.org/10.1174/021037008786140922

Patchin, J.W. y Hinduja, S. (2006). Bullies beyond the schoolyard: A preliminary look at cyberbullying. Youth Violence and Juvenile Justice, 4(2), 148-169. http://dx.doi. org/10.1177/1541204006286288

Patchin, J.W. y Hinduja, S. (2010). Cyberbullying and self-esteem. Journal of Schoo Health, 80(12), 614-620. http://dx.doi.org/10.1111/ j.1746-1561.2010.00548.x

Resett, S.A., Costa, D., Murata, C. y Falcone, N. (2015). Equivalencia factorial del Cuestionario de Agresores / Víctimas de Olweus según género. Interdisciplinaria, Revista de Psicología y Ciencias Afines, 32(1), 169-181. http://dx.doi.org/10.16888/interd.2015.32.1.9
Roberts, N., Axas, N., Nesdole, R. y Repetti, L. (2016). Pediatric emergency department visits for mental health crisis: Prevalence of cyber-bullying in suicidal youth. Child and Adolescent Social Work Journal, 33, 469-472. http://dx.doi.org/10.1007/s10560-016-0442-8

Sarudiansky, M. (2016). Análisis temático sobre la fibromialgia en la prensa escrita Argentina: descripciones generales y el rol de la psicología. Interface (Botucatu), 20(56), 25-36. http:// dx.doi.org/10.1590/1807-57622015.0008

Schudson, M. (2003): The Sociology of News. New York: W.W. Norton \& Company.

Shaw, M. (2005, 21 de enero). Bullies film fights by phone. The Times, p. 3. Recuperado de https://www.tes.com/news/tes-archive/tes-publication/bullies-film-fights-phone

Slonje, R., Smith, P.K. y Frisén, A. (2013). The nature of cyberbullying, and strategies for prevention. Computers in Human Behavior, 29(1), 26-32. http://dx.doi.org/10.1016/j. chb.2012.05.024

Smith, P.K., Mahdavi, J., Carvalho, M., Fisher, S., Russell, S. y Tippett, N. (2008). Cyberbullying: Its nature and impact in secondary school pupils. Journal of Child Psychology and Psychiatry and Allied Disciplines, 49(4), 376-385. http://dx.doi.org/10.1111/j.14697610.2007.01846.x

Tokunaga, R.S. (2010). Following you home from school: A critical review and synthesis of research on cyberbullying victimization. Computers in Human Behavior, 26(3), 277-287. http://dx.doi.org/10.1016/j.chb.2009.11.014

Van Geel, M., Vedder, P. y Tanilon, J. (2014). Relationship between peer victimization, cyberbullying, and suicide in children and adolescents. JAMA Pediatrics, 168(5), 435-442. http:// dx.doi.org/10.1001/jamapediatrics.2013.4143

Wachs, S., Jiskrova, G.K., Vazsonyi, A.T., Wolf, K.D. y Junger, M. (2016). A cross-national study of direct and indirect effects of cyberbullying on cybergrooming victimization via self-esteem. Psicología Educativa, 22(1), 61-70. http://dx.doi.org/10.1016/j.pse.2016.01.002

Willard, N.E. (2005). Cyberbullying and Cyberthreats. Recuperado de http://bcloud.marins- 
chools.org/SafeSchools/Documents/BP-CyberBandT.pdf

Willard, N.E. (2007). Cyberbullying and cyberthreats: Responding to the challenge of online social aggression, threats, and distress. Illinois: Research Press.

Wong-Lo, M. y Bullock, L.M. (2014). Digital metamorphosis: Examination of the bystander culture in cyberbullying. Aggression and Violent Behavior, 19(4), 418-422. http://dx.doi. org/10.1016/j.avb.2014.06.007

Ybarra, M.L. (2004). Linkages between depressive symptomatology and Internet harassment among young regular Internet users. Cyberpsychology and Behavior, 7(2), 247-257. http:// dx.doi.org/10.1089/109493104323024500

Recibido: 31 de enero de 2018 Aceptado: 26 de diciembre de 2019 